\title{
EXTRACCIÓN DE PETRÓLEO EN SUELO CONTAMINADO EMPLEANDO RAMNOLÍPIDOS PRODUCIDOS POR Pseudomonas aeruginosa ORA9
}

\author{
Javier Ernesto VILASÓ CADRE ${ }^{1 *}$, Odalys RODRÍGUEZ GÁMEZ² y Arelis ÁBALOS RODRÍGUEZ ${ }^{2}$
}

${ }^{1}$ Facultad de Ciencias Naturales y Exactas, Departamento de Química. Universidad de Oriente. Avenida Patricio Lumumba, Altos de Quintero, Santiago de Cuba, Cuba, C. P. 90600

${ }^{2}$ Facultad de Ciencias Naturales y Exactas, Centro de Estudios de Biotecnología Industrial, Universidad de Oriente. Avenida Patricio Lumumba, Altos de Quintero, Santiago de Cuba, Cuba, C. P. 90600

*Autor para correspondencia: javiere@uo.edu.cu

(Recibido noviembre 2015; aceptado diciembre 2016)

Palabras clave: remediación, hidrocarburos, diseño Box-Behnken

\section{RESUMEN}

La remoción de hidrocarburos empleando ramnolípidos ha mostrado potencialidades para su aplicación en tecnologías de remediación de ecosistemas impactados, así como en el aumento de la eficiencia de extracción de petróleo en pozos. En esta investigación se evaluó la remoción de hidrocarburos totales de petróleo de un suelo contaminado empleando los ramnolípidos producidos por Pseudomonas aeruginosa ORA9. Se realizó un estudio de estabilidad de los ramnolípidos considerando los factores temperatura, $\mathrm{pH}$ y fuerza iónica. Los ramnolípidos fueron estables en las condiciones ensayadas. Se utilizó un diseño Box-Behnken para evaluar la influencia de la temperatura, $\mathrm{pH}$ y tiempo de contacto, en la remoción de petróleo de suelo contaminado. Los tres factores influyeron significativamente, incrementándose la extracción al aumentar la temperatura y el tiempo de contacto y disminuir el pH. La máxima extracción alcanzada fue de un $75 \%$.

Key words: remediation, hydrocarbons, Box-Behnken design

\begin{abstract}
Hydrocarbon removal using rhamnolipids has shown potential for use in remediation technologies of impacted ecosystems as well as increasing the efficiency of oil extraction wells. In this research, the removal of total petroleum hydrocarbons in contaminated soil was evaluated using rhamnolipids produced by Pseudomonas aeruginosa ORA9. A stability study of rhamnolipids considering temperature, $\mathrm{pH}$ and ionic strength factors was performed. The rhamnolipids were stable in the tested conditions. A Box-Behnken design was employed to evaluate the influence of temperature, $\mathrm{pH}$ and contact time on the removal of petroleum from contaminated soil. All factors have a significative influence on the extraction of total petroleum hydrocarbons, increasing with temperature and contact time, and decreasing with $\mathrm{pH}$. The maximum extraction was $75 \%$.
\end{abstract}




\section{INTRODUCCIÓN}

Los ramnolípidos son biomoléculas superficialmente activas producidas por varias especies de bacterias del género Pseudomonas, con la mejor expresión en Pseudomonas aeruginosa (Muthusamy et al. 2008). Consisten estructuralmente en la unión de una o dos moléculas de ramnosa con una cadena de uno o dos ácidos $\beta$-hidroxicarboxílicos. Los ramnolípidos, junto a otro grupo de biocompuestos tensoactivos producidos por bacterias y hongos, son conocidos como biosurfactantes. Los ramnolípidos son completamente biodegradables, de baja toxicidad, se obtienen biotecnológicamente a partir de residuales, son estables y muestran propiedades superficiales superiores a los tensoactivos de origen sintético, los cuales, además de dominar el mercado, han mostrado ser persistentes en el ambiente, tóxicos y no sustentables por ser petroderivados (Abdel et al. 2010).

Aunque los ramnolípidos muestran cada vez más potencialidades para su introducción como competencia ante los tensoactivos sintéticos, su producción aún no es viable económicamente, por lo que sus aplicaciones han sido enfocadas fundamentalmente hacia sectores donde los sintéticos por las propias desventajas mencionadas, no pueden emplearse (Muthusamy et al. 2008). La industria petrolera es la que más terreno tecnológico exhibe para la introducción de estos biocompuestos, pues varios estudios han demostrado que la extracción de hidrocarburos empleando tensoactivos de origen biológico es un proceso eficiente, por lo que podrían ser empleados en tecnologías que solucionen dos de los principales problemas del sector industrial: la contaminación por hidrocarburos y la eficiencia de extracción (Raiger y López 2009, Hallmann y Medrzycka 2015, Pathaka y Pranav 2015, Zhang 2015).

La remediación basada en la extracción con tensoactivos puede ser aplicada tanto en suelos como en aguas contaminadas, aunque se han enfocado principalmente hacia los primeros ecosistemas (Lai et al. 2009, Hallmann 2015). Urum et al. (2006) mostraron la eficiencia de los ramnolípidos en la extracción de hidrocarburos del suelo, siendo ventajosos con respecto a tensoactivos de origen sintético que dominan el mercado, de forma similar, Yin et al. (2008) han demostrado que los ramnolípidos son mejores para la remediación de efluentes contaminados con crudo que los tensoactivos comerciales Tween y Tritón X-100. Guo y Mulligan (2006) demostraron que los ramnolípidos son capaces de remover más del $90 \%$ del estireno adsorbido en una mezcla compuesta por $66.6 \%$ de tierra y $33.3 \%$ de arena. Por otra parte, la extracción asistida de hidrocarburos es una tecnolo- gía que permite la recuperación más eficiente en los pozos de extracción (Sen 2008). La biodegradabilidad de los ramnolípidos los convierte en productos idóneos para su empleo en estos procesos.

La aplicación de los ramnolípidos en procesos de remediación, así como en la extracción asistida de crudo exige de compuestos estables ante condiciones como la temperatura, el $\mathrm{pH}$ y la salinidad. Los ecosistemas contaminados por hidrocarburos se localizan fundamentalmente en zonas costeras, por lo que es importante tener en cuenta la influencia de la salinidad en la actividad superficial del biosurfactante. Igualmente, el pH y la temperatura se vuelven importantes teniendo en cuenta la variabilidad de estas condiciones entre un ecosistema y otro. Por su parte, la extracción de crudo se realiza bajo condiciones extremas de temperatura. Por ello, con base en posibles aplicaciones, se realizan estudios de estabilidad de los biosurfactantes ante condiciones químicas y físicas específicas (Raiger y López 2009).

En este trabajo se muestran los resultados de ensayos de extracción de hidrocarburos totales de petróleo en muestras de suelo contaminado empleando ramnolípidos producidos por Pseudomonas aeruginosa ORA9. Previamente a los ensayos se realizó un estudio de estabilidad de los ramnolípidos teniendo en cuenta la tensión superficial ante cambios de temperatura, $\mathrm{pH}$ y fuerza iónica.

\section{MATERIALES Y MÉTODOS}

\section{Biosurfactante empleado}

Se utilizó la mezcla de ramnolípidos producidos durante el cultivo de Pseudomonas aeruginosa ORA9 en un medio mineral (Ramsay et al. 1990), empleando aceite de soja frito al $2 \%$ como única fuente de carbono. En todos los experimentos se utilizó el medio de cultivo libre de células obtenido por centrifugación (10 $000 \mathrm{rpm}, 10 \mathrm{~min}$ ). La concentración de ramnolípidos se determinó mediante el método espectrofotométrico, validado para la determinación en un medio de cultivo (Rodríguez et al. 2013).

\section{Estudio de estabilidad}

Se estudió la influencia de la temperatura, el pH y la fuerza iónica sobre la tensión superficial del sobrenadante libre de células con una concentración de ramnolípidos de $22.5 \mathrm{mg} / \mathrm{L}$. Para medir la tensión superficial se empleó un tensiómetro de anillo de $\mathrm{Du}$ Noüy (Krüss, Alemania), con un error instrumental de $1 \mathrm{mN} / \mathrm{m}$. Cada medición de la tensión superficial se realizó cinco veces. 


\section{Influencia de la temperatura}

El sobrenadante que contiene la mezcla de ramnolípidos se expuso a $20,50,70$ y $100^{\circ} \mathrm{C}$ durante $10 \mathrm{~min}$ previa determinación de la tensión superficial. Una vez alcanzada la temperatura del ensayo, se dejó enfriar hasta temperatura ambiente y se midió nuevamente la tensión superficial.

\section{Influencia del pH}

Se ajustó el $\mathrm{pH}$ de cuatro muestras de sobrenadante hasta 4, 7, 9 y 12 unidades respectivamente y posteriormente se midió la tensión superficial.

\section{Influencia de la fuerza iónica}

Se prepararon soluciones de 5, 15, 50 y $100 \mathrm{~g} / \mathrm{L}$ de $\mathrm{NaCl}$. La sal fue disuelta en el sobrenadante que contiene la mezcla de ramnolípidos y posteriormente se midió la tensión superficial.

\section{Ensayos de remoción de petróleo en suelo conta- minado}

Para la realización de los ensayos de remoción de hidrocarburos totales del petróleo se emplearon muestras de un suelo contaminado con petróleo ( $0.12 \mathrm{~g}$ de hidrocarburos/g de suelo) de la zona industrial de Santiago de Cuba. El suelo se tamizó a través de una malla de $2 \mathrm{~mm}$. Se pesó $1 \mathrm{~g}$ de suelo, se transfirió a un matraz Erlenmeyer y se añadieron $4 \mathrm{~mL}$ de medio de cultivo libre de células, con una concentración de ramnolípidos de $332 \mathrm{mg} / \mathrm{L}$, se agitó a $200 \mathrm{rpm}$ y luego se decantó. La fracción de petróleo presente en el suelo no emulsionada por el biosurfactante $\left(\mathrm{Hs}_{\text {no extraídos }}\right)$ se extrajo con diclorometano. Luego de evaporar el solvente se pesó el residuo. El petróleo total presente en las muestras de suelo contaminado ( $\mathrm{Hs}_{\text {en el suelo }}$ ) se determinó por extracción con el mismo solvente (Urum et al. 2004, modificado). El porcentaje de extracción se calculó según la ecuación 1.

$\% \mathrm{Hs}_{\text {extraídos }}=\frac{\mathrm{Hs}_{\text {en el suelo }}-\mathrm{Hs}_{\text {no extraidos }}}{\mathrm{Hs}_{\text {en el suelo }}} \cdot 100$

\section{Diseño de experimentos}

Se utilizó un diseño de experimentos de superficie de respuesta de tipo Box-Behnken (Agarry y Ogunleye 2012) para determinar el efecto de tres factores: temperatura, $\mathrm{pH}$ y tiempo de contacto en el proceso de extracción. Se realizaron 15 experimentos que incluyeron tres réplicas en el punto central para evaluar el error experimental (Cuadro I).
CUADRO I. FACTORES Y NIVELES ESTUDIADOS EN EL DISEÑO BOX-BEHNKEN PARA LA EXTRACCIÓN DE HIDROCARBUROS EN SUELO CONTAMINADO EMPLEANDO RAMNOLÍPIDOS ORA9

\begin{tabular}{lrcc}
\hline Factor & \multicolumn{3}{c}{ Niveles } \\
\cline { 2 - 4 } & -1 & 0 & 1 \\
\hline Temperatura $\left({ }^{\circ} \mathrm{C}\right)$ & 15 & 32.5 & 50 \\
pH & 4 & 7 & 10 \\
Tiempo de contacto (min) & 30 & 45 & 60 \\
\hline
\end{tabular}

\section{Procesamiento estadístico}

Los resultados fueron el promedio de tres mediciones independientes. El efecto de cada factor estudiado $(\mathrm{pH}$, temperatura y tiempo de contacto) se determinó mediante un análisis de varianza (ANDEVA). Por un análisis de regresión se obtuvo el modelo matemático que describe el comportamiento de los factores sobre la variable respuesta (porcentaje de remoción de petróleo). Se utilizó un nivel de significación $(\alpha)$ del 0.05 . Los análisis y los gráficos se realizaron con el programa estadístico Statgraphics Centurión XV 15.2.14.

\section{RESULTADOS}

\section{Estudio de estabilidad}

La concentración micelar crítica (CMC) de los ramnolípidos en el medio libre de células fue $87.47 \mathrm{mg} / \mathrm{L}$ para una tensión superficial de $39 \pm 0.5 \mathrm{mN} / \mathrm{m}$ y una tensión interfacial frente a queroseno de $5 \pm 0.5 \mathrm{mN} / \mathrm{m}$. La tensión superficial de la mezcla de ramnolípidos empleada en el estudio de estabilidad fue $44 \mathrm{mN} / \mathrm{m}$. Estos resultados se determinaron a $27^{\circ} \mathrm{C}$, pH 6.8 y la concentración de sales del medio de cultivo.

\section{Influencia de la temperatura}

La figura 1 muestra la tensión superficial del sobrenadante libre de células ante las temperaturas de experimentación. Se observan valores similares de tensión superficial entre cada temperatura ensayada, no existiendo diferencias estadísticamente significativas $(p=0.13, \alpha=0.05)$ entre las tensiones superficiales. Esto demuestra que los ramnolípidos son estables ante cambios de temperatura en el rango estudiado.

\section{Influencia del pH}

La figura 2 muestra la tensión superficial ante los valores de $\mathrm{pH}$ ensayados durante el estudio de estabilidad. Los valores de tensión superficial son similares. 


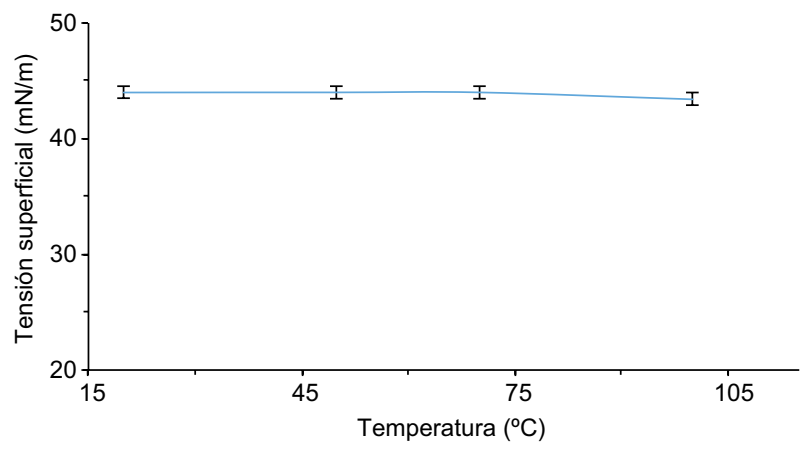

Fig. 1. Tensión superficial del medio de cultivo libre de células con $22.5 \mathrm{mg} / \mathrm{L}$ de ramnolípidos luego de la exposición a diferentes temperaturas

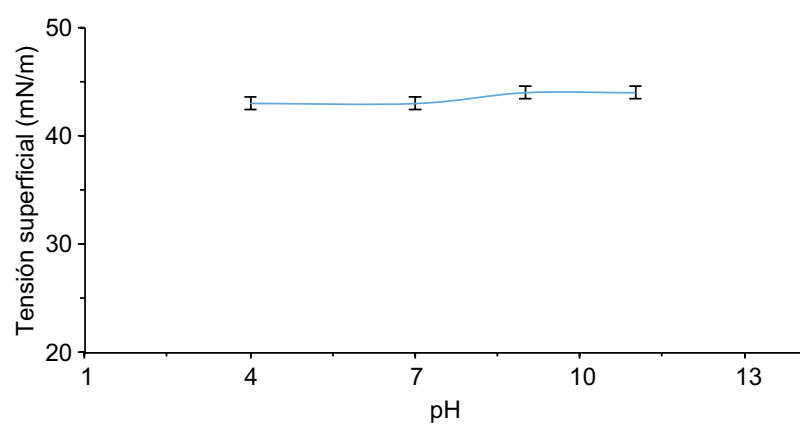

Fig. 2. Tensión superficial del medio de cultivo libre de células con $22.5 \mathrm{mg} / \mathrm{L}$ de ramnolípidos a diferentes valores de $\mathrm{pH}$

El ANDEVA arrojó un valor p (0.0876) superior al nivel de significación (0.05), evidenciando que no existen diferencias estadísticamente significativas

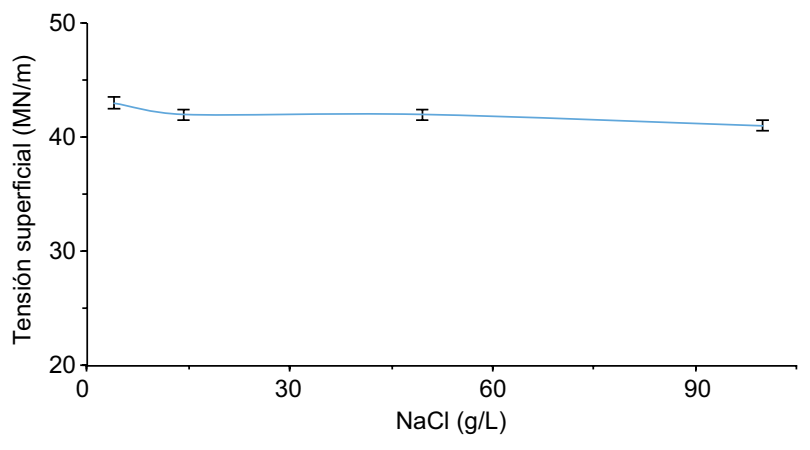

Fig. 3. Tensión superficial del medio de cultivo libre de células con $22.5 \mathrm{mg} / \mathrm{L}$ de ramnolípidos a diferentes concentraciones de cloruro de sodio $(\mathrm{NaCl})$

entre las tensiones superficiales y demostrando la estabilidad de los ramnolípidos ante los cambios de $\mathrm{pH}$.

\section{Influencia de la fuerza iónica}

La figura 3 muestra la tensión superficial a las diferentes concentraciones de $\mathrm{NaCl}$ ensayadas. En este caso, el ANDEVA arrojó un valor p (0.0018) inferior al nivel de significancia (0.05), evidenciando que existen diferencias estadísticamente significativas entre las tensiones superficiales a cada concentración de $\mathrm{NaCl}$.

\section{Remoción de hidrocarburos totales de petróleo en suelo contaminado}

El cuadro II muestra los experimentos de remoción de petróleo del diseño Box-Behnken con los correspondientes resultados.

\begin{tabular}{|c|c|c|c|c|c|}
\hline \multirow[t]{2}{*}{ Experimento } & \multirow{2}{*}{$\begin{array}{c}\text { Temperatura } \\
\left({ }^{\circ} \mathrm{C}\right)\end{array}$} & \multirow[t]{2}{*}{$\mathrm{pH}$} & \multirow{2}{*}{$\begin{array}{l}\text { Tiempo de } \\
\text { contacto } \\
\text { (min) }\end{array}$} & \multicolumn{2}{|c|}{ Extracción (\%) } \\
\hline & & & & Observado & Predicho \\
\hline 1 & 15 & 4 & 45 & 64 & 61.9 \\
\hline 2 & 50 & 7 & 30 & 55 & 55.1 \\
\hline 3 & 50 & 4 & 45 & 69 & 68.8 \\
\hline 4 & 32.5 & 4 & 30 & 55 & 55 \\
\hline 5 & 32.5 & 7 & 45 & 53 & 51 \\
\hline 6 & 32.5 & 7 & 45 & 44 & 51 \\
\hline 7 & 32.5 & 10 & 30 & 49 & 46.7 \\
\hline 8 & 50 & 7 & 60 & 65 & 62.8 \\
\hline 9 & 32.5 & 4 & 60 & 75 & 77.2 \\
\hline 10 & 32.5 & 10 & 60 & 48 & 48 \\
\hline 11 & 15 & 7 & 30 & 36 & 38.1 \\
\hline 12 & 32.5 & 7 & 45 & 56 & 51 \\
\hline 13 & 15 & 7 & 60 & 54 & 53.9 \\
\hline 14 & 50 & 10 & 45 & 54 & 56.1 \\
\hline 15 & 15 & 10 & 45 & 37 & 37.1 \\
\hline
\end{tabular}


CUADRO III. ANÁLISIS DE VARIANZA DEL DISEÑO BOX-BEHNKEN PARA LA EXTRACCIÓN DE HIDROCARBUROS EN UN SUELO CONTAMINADO EMPLEANDO RAMNOLÍPIDOS ORA9

\begin{tabular}{lcccrc}
\hline Fuente & S.C. & g.l. & C.M. & Cociente F & Valor p \\
\hline A:Temperatura & 338.0 & 1 & 338.0 & 15.91 & 0.0104 \\
B: pH & 703.125 & 1 & 703.125 & 33.09 & 0.0022 \\
C: Tiempo & 276.125 & 1 & 276.125 & 12.99 & 0.0155 \\
AA & 0.519231 & 1 & 0.519231 & 0.02 & 0.8819 \\
AB & 36.0 & 1 & 36.0 & 1.69 & 0.2498 \\
AC & 16.0 & 1 & 16.0 & 0.75 & 0.4252 \\
BB & 78.9808 & 1 & 78.9808 & 3.72 & 0.1118 \\
BC & 110.25 & 1 & 110.25 & 5.19 & 0.0717 \\
CC & 4.67308 & 1 & 4.67308 & 0.22 & 0.6588 \\
Error Total & 106.25 & 5 & 21.25 & & \\
Total & 1666.93 & 14 & & & \\
\hline
\end{tabular}

S.C. = suma de cuadrados, g.l. = grados de libertad, C.M.= cuadrado medio

El cuadro III muestra el ANDEVA del diseño experimental propuesto para la remoción de hidrocarburos mostrado en el cuadro II.

Para el intervalo de confianza de un $95 \%$, el valor $\mathrm{p}$ de cada uno de los factores principales (temperatura, $\mathrm{pH}$ y tiempo de contacto) es inferior al nivel de significación $(\alpha=0.05)$. Por tanto, los tres factores tienen influencia estadísticamente significativa sobre la remoción de hidrocarburos totales de petróleo. Las interacciones entre los factores principales no muestran valores $\mathrm{p}$ inferiores a $\alpha$, por lo que no influyen significativamente.

El ajuste de los resultados, mediante un análisis de regresión, para describir la relación entre el porcentaje de remoción y las tres variables estudiadas está dado por la ecuación 2:

\%Remoción $=51.0+6.5 \times$ Temp -

$9.375 \times \mathrm{pH}+5.875 \times \mathrm{t}$ de contacto

El coeficiente de determinación $\left(\mathrm{R}^{2}\right)$ fue 0.936 , mientras que el coeficiente de determinación ajustado para grados de libertad $\left(\mathrm{R}^{2}\right.$ adj. $)$ fue 0.821 .

La figura 4 muestra el gráfico de efectos principales. Al aumentar la temperatura y el tiempo de contacto en agitación, aumenta la extracción de hidrocarburos, en cambio, al aumentar el $\mathrm{pH}$ disminuye el resultado de la extracción.

\section{DISCUSIÓN}

La estabilidad de los ramnolípidos hasta los $100{ }^{\circ} \mathrm{C}$ ha sido comprobada por otros autores. Thaniyavarn et al. (2006) en un estudio de estabilidad similar,

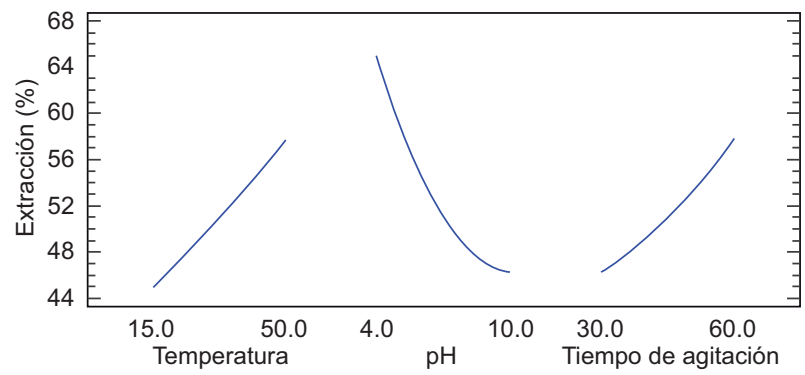

Fig. 4. Efectos principales para la extracción de hidrocarburos en un suelo contaminado empleando ramnolípidos

reportan ramnolípidos producidos por Pseudomonas aeruginosa $\mathrm{A} 41$ estables hasta los $100^{\circ} \mathrm{C}$, teniendo en cuenta la tensión superficial. Los ramnolípidos producidos por Pseudomonas aeruginosa UCP0992 mostraron estabilidad durante dos horas a $90{ }^{\circ} \mathrm{C}$ (Silva et al. 2010). La temperatura máxima evaluada en este trabajo fue $100^{\circ} \mathrm{C}$, sin embargo, se ha demostrado que los ramnolípidos pueden ser estables a mayores temperaturas. Así, los producidos por Pseudomonas fluorescens fueron estables de 25 a $120{ }^{\circ} \mathrm{C}$ (Abouseoud et al. 2008).

Los ramnolípidos estudiados en este trabajo son estables en un amplio rango de $\mathrm{pH}$ (4 a 11) en comparación con los resultados obtenidos por Pornsunthorntawee et al. (2008), quienes reportan la estabilidad de los ramnolípidos, producidos por $P$. aeruginosa SP4 para valores de $\mathrm{pH}$ entre 6 y 11 . Igualmente, los ramnolípidos caracterizados por Techaoei et al. (2011) fueron estables sólo en el rango de $\mathrm{pH}$ de 6 a 10. Kalimuthu et al. (2013) reportan una mezcla de ramnolípidos estables sólo hasta $\mathrm{pH} 10$. La estabilidad de los ramnolípidos hasta $\mathrm{pH} 4$ ha sido 
ampliamente reportada por otros autores (Anna Joice P. y Parthasarathi 2014, Kaya et al. 2014, Velmurugan et al. 2015). A partir de este valor se observa la protonación de los grupos carboxilato, provocando la precipitación del ramnolípido. La variación del valor de $\mathrm{pH}$ al cual ocurre la protonación está relacionado con la estructura molecular mayoritaria en la mezcla producida (Abdel et al. 2008, Shin et al. 2008).

La fuerza iónica de una disolución es directamente proporcional a la concentración de los iones de cada especie presente en una disolución, así como a la carga al cuadrado de estos. La fuerza iónica tiene influencia en la solubilidad y en otras propiedades de los electrólitos. La tensión superficial depende de la fuerza iónica, debido a que al aumentar esta, disminuye la concentración efectiva de tensoactivo. Cuando un electrólito se encuentra en alta concentración en una solución, la fuerza iónica se debe fundamentalmente a ese electrolito. Por ello una forma de ajustar la fuerza iónica es garantizando una alta concentración de una sal fuerte y soluble como el $\mathrm{NaCl}$ (Shaw 1977).

El ANDEVA para la comparación de las tensiones superficiales mostradas en cada concentración arrojó diferencias estadísticamente significativas, sin embargo, teniendo en cuenta el amplio rango de concentración de $\mathrm{NaCl}$ (5 a 100 g/L) evaluado, y que la diferencia máxima entre los valores es de $3 \mathrm{mN} / \mathrm{m}$, para un error instrumental de $1 \mathrm{mN} / \mathrm{m}$, es posible afirmar que desde el punto de vista químico y físico esta variación no se considera significativa. Pirollo et al. (2008) reportan la estabilidad de la tensión superficial hasta los $35 \mathrm{~g} / \mathrm{L}$ de $\mathrm{NaCl}$. Con resultados similares, Abdel et al. (2009) muestran la actividad superficial estable hasta los $30 \mathrm{~g} / \mathrm{L}$ para una variación de la tensión superficial desde los 33 hasta los $37 \mathrm{mN} / \mathrm{m}$ de una disolución de $50 \mathrm{~g} / \mathrm{L}$ de ramnolípidos purificados. Estos resultados de estabilidad son inferiores al obtenido para los ramnolípidos producidos por Pseudomonas aeruginosa ORA9, no obstante, Abouseoud et al. (2008) reportan la estabilidad de la tensión superficial de una solución de ramnolípidos purificados hasta los $150 \mathrm{~g} / \mathrm{L}$ de cloruro de sodio en el medio.

De manera general, los resultados del estudio de estabilidad mostraron ramnolípidos estables ante cambios de temperatura, $\mathrm{pH}$ y fuerza iónica. Los resultados del estudio son básicos para la evaluación de la remoción de petróleo empleando los ramnolípidos considerando diferentes condiciones físicas y químicas.

Los resultados del diseño de experimentos muestran que, al aumentar la temperatura aumenta con significación estadística la extracción de los hidrocarburos. Este comportamiento se asocia al hecho de que la tensión interfacial del sistema petróleo agua disminuye significativamente debido a la presencia de ramnolípidos, (Patel y Desai 1997, Lovaglio et al. 2011, Pirog et al. 2015) facilitando la emulsificación de los hidrocarburos. El aumento de la temperatura disminuye la tensión interfacial, lo que favorecerá desde el punto de vista termodinámico la formación de una emulsión (Shaw 1977). El comportamiento está en correspondencia con los resultados reportados por Urum et al. (2003 y 2004). Bordoloi y Konwar (2008), utilizando los biosurfactantes producidos por las cepas de P. aeruginosa MTCC7815, MTCC7812 y MTCC 8165 , muestran porcentajes de remoción máximos de hidrocarburos en columnas de arena saturadas que van desde un 54 hasta un $90 \%$ desde temperatura ambiente hasta $90{ }^{\circ} \mathrm{C}$ respectivamente, demostrando que, al aumentar la temperatura aumenta la extracción de los hidrocarburos.

Los tensoactivos aniónicos se encuentran más disociados en medio básico debido a la desprotonación de los grupos hidrofílicos, esto favorece la formación de un mayor número de micelas al pasar de medio ácido o neutro a básico (Shaw 1977). Sin embargo, en este estudio a medida que se acidifica el medio, dentro de los límites establecidos en el diseño experimental, la extracción de hidrocarburos es mayor. Shin et al. (2008) han demostrado que los ramnolípidos forman diferentes tipos de agregados moleculares al variar el $\mathrm{pH}$. A pH 4 se ha observado la formación de estructuras en forma de hojas laminares. A pH 5 además de las hojas laminares, aparecen vesículas. Entre 4 y 5 unidades de $\mathrm{pH}$ los agregados moleculares descritos son de gran tamaño. A valores de $\mathrm{pH} 6$ predominan las vesículas, formándose también micelas. Cuando se alcanza el pH 7 solamente se detectan micelas. Las estructuras formadas en dependencia del $\mathrm{pH}$, su estabilidad y tamaño están en correspondencia con el nivel de protonación de los grupos carboxilato de los ramnolípidos. Similares resultados, pero con menores especificaciones habían sido descritos por Champion et al. (1995), quienes observaron la diminución del tamaño de los agregados moleculares de ramnolípidos al variar el $\mathrm{pH}$ desde 5.5 hasta 8 , donde se forman hojas laminares, pasando por vesículas $\mathrm{y}$, finalmente, al $\mathrm{pH}$ superior, micelas. La dispersión de moléculas orgánicas, tales como los hidrocarburos, será más favorable en un tipo u otro de los agregados moleculares en dependencia del tamaño de las moléculas de la fase dispersa. Así, la dispersión de hidrocarburos poliaromáticos será más favorable en hojas laminares y vesículas, mientras 
que los hidrocarburos de menor tamaño molecular, tales como los n-alcanos inferiores, serán dispersados más fácilmente por micelas (Shin et al. 2008).

Los resultados obtenidos por Zhang (2015) demostraron que con el incremento de la alcalinidad de la solución de ramnolípidos, aumentaba la eficiencia de remoción de los hidrocarburos aromáticos policíclicos en suelos contaminados con petróleo. Shin et al. (2008) reportan una mayor dispersión de fenantreno a valores menores de $\mathrm{pH}$ hasta 4 , a partir de este valor observaron adsorción de las moléculas del ramnolípido a las partículas de suelo. El pH de mayor extracción fue 5. Por otra parte, el $\mathrm{pH}$, además de modificar las características morfológicas de los agregados moleculares de ramnolípidos, también tiene influencia en la desadsorción de los hidrocarburos del suelo y, por tanto, en su mayor disponibilidad para la emulsificación y posterior extracción (Dahn y Reyes 1992).

El aumento de la extracción con el tiempo de contacto está asociado a que la agitación mecánica es uno de los factores a considerar en la dispersión de una sustancia en el seno de otra. Además, la agitación influye en los fenómenos de desadsorción de las partículas adsorbidas al suelo.

Otros autores reportan estudios de extracción de petróleo en suelos empleando ramnolípidos. Urum et al. (2003) reportan la remoción de un $80 \%$ de hidrocarburos de petróleo a los 10 min de agitación a $50^{\circ} \mathrm{C}$ y empleando $15 \mathrm{~mL}$ de solución de ramnolípidos al $0.5 \%$. El resultado es cercano a la extracción máxima $(75 \%)$ obtenida a una temperatura de $32.5{ }^{\circ} \mathrm{C}$, pH 4 y un tiempo de contacto de 60 min (Cuadro II), sin embargo, la temperatura de extracción en esta investigación es menor, lo cual es ventajoso. Estos autores evaluaron además, bajo condiciones óptimas, otras biomoléculas superficialmente activas, la aescina y una lecitina, que mostraron un $30 \mathrm{y}$ un $10 \%$ de remoción respectivamente. Los resultados alcanzados por Urum et al. (2006), reflejan la remoción de un $44 \%$ de los hidrocarburos totales del petróleo en suelo empleando ramnolípidos. En el caso del dodecilsulfato de sodio la remoción fue de un $46 \%$, mientras que una saponina removió un $27 \%$. Por otra parte, Lai et al. (2009) muestran un $40 \%$ de remoción de hidrocarburos totales del petróleo en suelos contaminados empleando una solución de ramnolípidos de $500 \mathrm{mg} / \mathrm{L}$ a $25^{\circ} \mathrm{C}$. Nitschke et al. (2010) reportaron un $67 \%$ de remoción de crudo en este tipo de matriz empleando ramnolípidos al $0.1 \%$ a temperatura ambiente; mientras que Hallmann y Medrzycka (2015) reportan la remoción de los hidrocarburos del petróleo de un suelo contaminado a $23{ }^{\circ} \mathrm{C}$ empleando una mezcla de ramnolípidos puri- ficados en concentraciones desde 0.5 hasta $3 \mathrm{~g} / \mathrm{L}$, la extracción en ese rango fue desde el $67 \%$ a un $99 \%$ respectivamente. La menor concentración es comparable a la empleada en este estudio, y se observa similitud en el porciento de extracción. Los resultados de extracción dependerán, además de las condiciones experimentales, de las propiedades químicas y físicas del suelo y de los contaminantes. Urum et al. (2003) demuestran la influencia de las propiedades químicas y físicas de los suelos en los resultados de extracción de hidrocarburos del petróleo empleando varios tensoactivos, entre ellos, ramnolípidos.

\section{CONCLUSIONES}

Los ramnolípidos producidos por Pseudomonas aeruginosa ORA9 son estables ante variaciones de temperatura, $\mathrm{pH}$ y fuerza iónica. La extracción de petróleo fue máxima a una temperatura de $32.5^{\circ} \mathrm{C}$, un $\mathrm{pH}$ de 4 y un tiempo de contacto de $60 \mathrm{~min}$, alcanzándose el 75 \% de remoción.

\section{REFERENCIAS}

Abdel A.M., Lépine F. y Déziel E. (2010). Rhamnolipids: diversity of structures, microbial origins and roles. Appl. Microbiol. Biotechnol. 86 (5), 1323-1336.

DOI: $10.1007 / \mathrm{s} 00253-010-2498-2$

Abdel A. M., Mabrouk M. y Abdel N. (2009). Characterization of rhamnolipids produced by Pseudomonas aeruginosa isolate Bs20. Appl. Biochem. Biotechnol. 157 (2), 329-345. DOI: 10.1007/s12010-008-8285-1

Abouseoud M., Yataghene A., Amrane A. y Maachi R. (2008). Biosurfactant production by free and alginate entrapped cells of Pseudomonas fluorescens. J. Ind. Microbiol. Biotechnol. 35 (11), 1303-1308.

DOI: $10.1007 / \mathrm{s} 10295-008-0411-0$

Agarry S. E. y Ogunleye O. O. (2012). Box-Behnken design application to study enhanced bioremediation of soil artificially contaminated with spent engine oil using biostimulation strategy. Int. J. Energy Environ. Eng. 31, 1-14. DOI: 10.1186/2251-6832-3-31

Anna Joice P. y Parthasarathi R. (2014). Production and characterization of biosurfactant from Pseudomonas aeruginosa $\mathrm{PBSC} 1$ isolated from mangrove ecosystem. Afr. J. Biotechnol. 13 (33), 3394-3401. DOI: $10.5897 / \mathrm{AJB} 2014.13843$

Bordoloi N. K. y Konwar B. K. (2008). Microbial surfactant-enhanced mineral oil recovery under laboratory conditions. Colloids Surf. B Biointerfaces. 63 (1), 73-82. DOI: 10.1016/j.colsurfb.2007.11.006 
Champion J. T., Gilery J.C., Lamparski H., Petterer J. y Miller R.M. (1995). Electron microscopy of rhamnolipid (biosurfactant) morphology: Effect of $\mathrm{pH}$, cadmium, and octadecane. J. Colloid. Interface Sci. 170 (2), 569-574. DOI: 10.1006/jcis.1995.1136

Dahn C. J. y Reyes B. N. (1992). Soil remediation methods. Memorias. Twenty-Fifth DoD Explosives Safety Seminar. California, EUA. 13 al 15 de julio, 1992.

Guo Y. y Mulligan C.N. (2006). Combined treatment of styrene-contaminated soil by rhamnolipids washing followed by anaerobic treatment. En: Hazardous materials in soil and atmosphere. (R.C. Hudson, Ed.). Nova Science Publishers, Nueva York, EUA, pp. 1-38.

Hallmann E. y Medrzycka K. (2015). Wetting properties of biosurfactant (rhamnolipid) with synthetic surfactants mixtures in the context of soil remediation. Ann. Univ. Mariae Curie-Skłodowska. 70 (1), 29-39.

DOI: 10.1515/umcschem-2015-0003

Kalimuthu K., Ganesh S., Vimalan J. y Solomon R. (2013). Molecular and physicochemical characterization of rhamnolipids biosurfactant produced by Pseudomonas sp. JSK6. Memorias. International Conference of Biotechnology for Innovative Aplications. Kerala, India. 10 al 14 de agosto, 2013.

Kaya T., Aslim B. y Kariptaş E. (2014). Production of biosurfactant by Pseudomonas spp. isolated from industrial waste in Turkey. Turk J. Biol. 38, 307-317. DOI: 10.3906/biy-1303-18

Muthusamy K., Gopalakrishnan S., Kochupappy T. y Sivachidambaram P. (2008). Biosurfactants: Properties, commercial production and application. Current Sci. 94 (6), 736-747.

Lai C., Huang L., Wei Y. y Chang J. (2009). Biosurfactantenhanced removal of total petroleum hydrocarbons from contaminated soil. J. Hazard Mater. 167 (1-3), 609-614. DOI: 10.1016/j.jhazmat.2009.01.017

Lovaglio R., dos Santos F., Jafelicci M. y Contiero J. (2011). Rhamnolipid emulsifying activity and emulsion stability: $\mathrm{pH}$ rules. Colloids Surf. B: Biointerfaces. 85 (2), 301-305. DOI: 10.1016/j.colsurfb.2011.03.001

Nitschke M., Costa S. y Contiero J. (2010). Structure and applications of a rhamnolipid surfactant produced in soybean oil waste. Appl. Biochem. Biotechnol. 160 (7), 2066-2074. DOI: 10.1007/s12010-009-8707-8

Patel R.M. y Desai A.J. (1997) Surface-active properties of rhamnolipids from Pseudomonas aeruginosa GS3. J. Basic Microb. 37 (4), 281-286. DOI: $10.1002 /$ jobm.3620370407

Pathaka A.N. y Pranav H. (2015). Optimization of rhamnolipid: A new age biosurfactant from Pseudomonas aeruginosa MTCC 1688 and its application in oil recovery, heavy and toxic metals recovery. J. Bioprocess Biotech. 5, 1-29. DOI: 10.4172/2155-9821.1000229
Pirog T., Konon A. y Savenko I. (2015). Microbial surfactants in environmental technologies. Biotechnol. Acta 8 (4), 21-39. DOI: 10.15407/biotech8.04.021

Pirollo M. P. S, Mariano A.P., Lovaglio R.B., Costa S.G.V.A.O., Walter V., Hausmann R. y Contiero J. (2008). Biosurfactant synthesis by Pseudomonas aeruginosa LBI isolated from a hydrocarbon-contaminated site. J. Appl. Microbiol. 105 (5), 1484-1490.

DOI: $10.1111 / \mathrm{j} .1365-2672.2008 .03893 . \mathrm{x}$

Pornsunthorntawee O., Wongpanit P., Chavadej S., Abe M. y Rujiravanit R. (2008). Structural and physicochemical characterization of crude biosurfactant produced by Pseudomonas aeruginosa SP4 isolated from petroleum-contaminated soil. Bioresour. Technol. 99 (6), 1589-1595.

DOI: $10.1016 /$ j.biortech.2007.04.020

Raiger L. y López N. (2009). Los biosurfactantes y la industria petrolera. Química Viva 8 (3), 146-161.

Ramsay B., Lomaliza K., Chavarie C., Dube B., Batille P. y Ramsay J. (1990). Production of poly- $\beta$ hydroxybutyric- $\beta$-hydroxyvaleric acids. Appl. Environ. Microbiol. 56, 2093-2098.

Rodríguez O., Vilasó J.E., Aguilera I., Pérez R.M. y Ábalos A. (2013). Validación por verificación del método colorimétrico de la antrona para la cuantificación de ramnolípidos. Revista Cubana de Química 25 (3), 287-294.

Sen R. (2008). Biotechnology in petroleum recovery: The microbial EOR progress in energy and combustión. Prog. Energy Combust. Sci. 34 (6), 714-724. DOI: 10.1016/j.pecs.2008.05.001

Shin K., Kim K., Kim J., Lee K. y Hang S. (2008). Rhamnolipid morphology and phenanthrene solubility at different $\mathrm{pH}$ values. J. Environ. Qual. 37 (2), 509-514. DOI: $10.2134 /$ jeq2007.0258

Silva S.N. R.L., Farias C.B.B., Rufino R.D., Luna J.M. y Sarubbo L.A. (2010). Glycerol as substrate for the production of biosurfactant by Pseudomonas aeruginosa UCP0992. Colloids Surf. B Biointerfaces. 79 (1), 174-183.

DOI: $10.1016 /$ j.colsurfb.2010.03.050

Shaw D. (1977). Introducción a la química de superficies y coloides. Alhambra, Madrid, España, 275 pp.

Thaniyavarn J., Chongchin A., Wanitsuksombut N., Thaniyavarn S., Pinphanichakarn P., Leepipatpiboon N., Morikawa M. y Kanaya S. (2006). Biosurfactant production by Pseudomonas aeruginosa A41 using palm oil as carbon source. J. Gen. Appl. Microbiol. 54 (2), 215-222.

Techaoei S., Lumyong S. y Prathumpai W. (2011). Screening characterization and stability of biosurfactant produced by Pseudomonas aeruginosa SCMU106 isolated from soil in northern Thailand. Asian J. Biol. Sci. 4 (4), 340-351. DOI: 10.3923/ajbs.2011.340.351 
Urum K., Grigson S., Pekdemir T. y McMenamy S. (2006). A comparison of the efficiency of different surfactants for removal of crude oil from contaminated soils. Chemosphere. 62 (9), 1403-1410.

DOI: 10.1016/j.chemosphere.2005.05.016

Urum K., Pekdemir T. y Çopur M. (2003). Optimum conditions for washing of crude oil-contaminated soil with biosurfactant solutions. Process. Saf. Environ. 81 (3), 203-209. DOI: 10.1205/095758203765639906

Urum K., Pekdemir T. y Çopur M. (2004). Surfactants treatment of crude oil contaminated soils. J. Colloid Interf. Sci. 276 (2), 456-464.

DOI: $10.1016 /$ j.jcis.2004.03.057

Velmurugan M., Baskaran A., Kumar S., Sureka I., Arockia Raj E., Emelda J. y Sathiyamurthy K. (2015). Screening, stability and antibacterial potential of rhamnolipids from Pseudomonas sp., isolated from hydrocarbon contaminated soil. J. App. Pharm. Sci. 5 (8), 26-33. DOI: $10.7324 / \mathrm{JAPS} .2015 .50805$
Yin J., Qiang Y.J., Jia Y., Ye J., Peng H., Qin H., Zhang N. y He B. (2008). Characteristics of biosurfactant produced by Pseudomonas aeruginosa S6 from oil-containing wastewater. Proc. Biochem. 44 (3), 302-308. DOI: 10.1016/j.procbio.2008.11.003

Zhang W. (2015). Batch washing of saturated hydrocarbons and polycyclic aromatic hydrocarbons from crude oil contaminated soils using bio-surfactant. J. Cent. South Univ. 22 (3), 895-903.

DOI: $10.1007 / \mathrm{s} 11771-015-2599-2$

Zhang Y. y Miller R. M. (1992). Enhanced octadecane dispersion and biodegradation by a Pseudomonas rhamnolipid biosurfactant. Appl. Environ. Microbiol. 58 (10), 3276-3282. 